\title{
A molecular dynamics model to measure forces between cellulose fibril surfaces: on the effect of non- covalent polyelectrolyte adsorption
}

\author{
Carlos Sáenz Ezquerro (id) C Cristina Crespo Miñana (D) Salvador Izquierdo (D) \\ Manuel Laspalas
}

\begin{abstract}
This study describes the development of representative models of cellulose fibril surface (CFS) as a first approximation to the study of the molecular interactions that are developed between cellulose fibres. In order to assess its sensitivity and representativeness towards the main factors affecting the bonding properties at the fibre scale, these models were non-covalently surface modified with two types of polyelectrolytes, sodium carboxymethyl cellulose (CMC-ONa) and a cationic polyacrylamide (CPAM). From the analysis of pair correlation functions $(\mathrm{g}(\mathrm{r}))$ it was possible to assess the main interactions of adsorption of polyelectrolytes towards the (1-10) hydrophilic cellulose, which were due to electrostatic interactions coupled with hydrogen bonding. Besides, the bond strength between fibril surfaces through the (100) hydrophobic surface was calculated from pull
\end{abstract}

out simulations (using steered molecular dynamics). Using a rate of change of force of $0.159 \mathrm{nN} \mathrm{ps}^{-1}$, the calculated bond strength for the neat CFS model (nanometer scale) was two to three orders of magnitude higher than the experimental values observed at the fibre scale (micrometer scale). The results for the polyelectrolyte modified setups supported the validity of the CFS models to reproduce the expected behavior of inter-fibre joints in terms of the specific bond strength and the relative bonded area at the fibre scale in cellulose materials, and thereby the CFS models are a suitable complement, in conjunction with other techniques, for the systematic study of the effect (in qualitative terms) of chemical or physical factors on the bond strength properties of cellulosic materials.

Electronic supplementary material The online version of this article (https://doi.org/10.1007/s10570-018-2166-8) contains supplementary material, which is available to authorized users.

C. Sáenz Ezquerro $(\bowtie) \cdot C$. Crespo Miñana

S. Izquierdo · M. Laspalas

Aragon Institute of Technology ITAINNOVA, María de

Luna 7-8, 50018 Saragossa, Spain

e-mail: csaenz@itainnova.es 


\section{Graphical abstract}

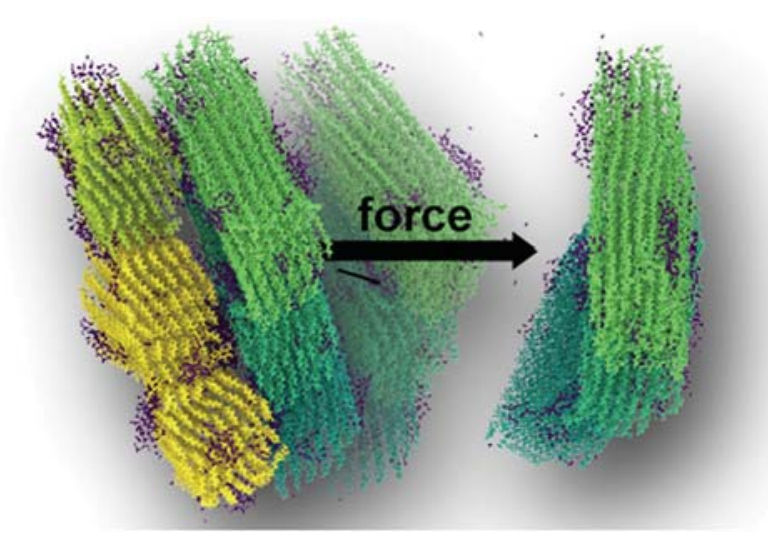

Keywords Molecular dynamics - Bond strength · Force $\cdot$ Polyelectrolyte $\cdot$ Adsorption $\cdot$ Pull out

\section{Introduction}

The properties of paper are largely dependent on the bonds between the fibres. To mention a few, properties such as strength, stiffness, opacity, smoothness, porosity, dimensional stability, density, and formation are influenced by the bonds between cellulose fibres (Torgnysdotter et al. 2007). Five mechanisms are considered to be responsible of the development of the fibre-fibre joint: (i) mechanical interlocking, (ii) hydrogen bonds, (iii) electrostatic interactions, (iv) interdiffusion of molecules and (v) induced dipoles (Lindström et al. 2005). Nowadays it is yet a controversial fact what is the prevalent mechanism (Schmied et al. 2012), although recent works that has attempted to quantify the contribution of each different bonding mechanism has identified van der Waals forces, Coulomb bonding and the extent of molecular contact as the key factors for fibre-fibre bonding, above even hydrogen bonding (Hirn and Schennach 2015), usually considered as the most relevant mechanism.

The measurement of the strength or force needed to separate fibre-fibre joints becomes essential to understand the behavior of paper and its relationship with fibre properties. In paper physics there are two common types of methods for measuring this property: indirectly, by mechanical testing of whole sheets (Nordman 1957); or directly testing individual fibre to

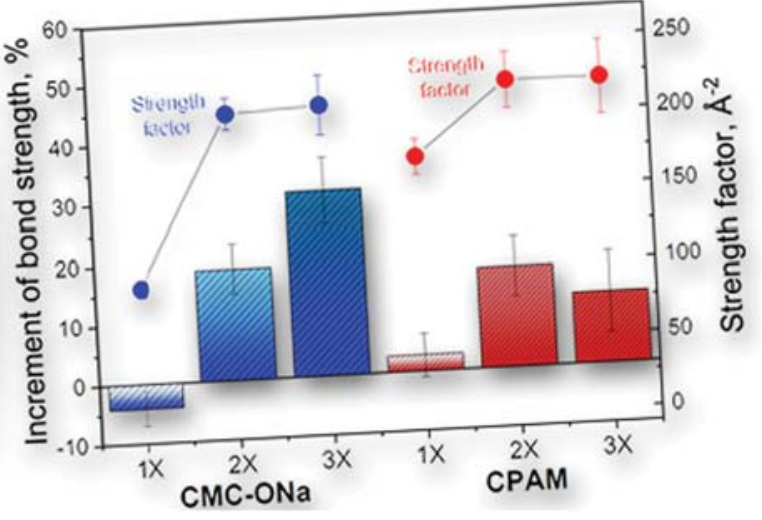

fibre cross structures (Fischer et al. 2012; Schmied et al. 2012; Magnusson et al. 2013). The advantage and attractiveness of indirect measurements, is that several joints are measured simultaneously and an average result is obtained, and fibre joints are in the same state as in the final material. Nevertheless, this method is controversial since some intrafibre breakage is also produced during straining of the sheet (Page 2002), affecting the measured irreversible work and obtained bond strength. On the other hand, direct experimental measurements of the strength between fibres requires of special equipment and careful manipulation of fibres to prepare adequate fibre-fibre joints. Typically, a fibre-fibre cross is prepared by fixing the supporting fibre to the sample holder while the loaded fibre, which crosses over the supported one, has one end fixed to a mobile device. However this method is not free of drawbacks, since the measured strength values will depend on both geometric and material properties of the two fibres (Schmied et al. 2012; Magnusson et al. 2013). Depending on setup, twisting of the loaded fibre could occur and thus give rise to a combined mode of loading (Schmied et al. 2012). In addition, fibre wall damage and failure at the $\mathrm{S} 1-\mathrm{S} 2$ layers interface is probably produced in the stronger bonds instead of failing at the fibre-fibre interface (Stratton 1993).

Together with experimentation, simulation has also been employed to study the fibre-fibre bond. Usually FEM methods have been employed to analyze the contribution of normal and shear components to the measured strength values, as well as indications about the order of magnitude of the errors introduced by the initial geometry or rotations of fibres (Magnusson and 
Östlund 2013). FEM models require experimental measurements to fit their parameters in order to adjust behavior of simulations to real materials, but the attractiveness of simulation methods would lie on determining the fibre bond strength directly without any previous experimentation. Usually this fact would imply downing to scales based on fundamental laws such as atomistic or other coarse-grained ones using molecular dynamics (MD) simulations (Alder and Wainwright 1959) since, as was previously pointed out, fibre bond properties are dependent on several molecular mechanisms (e.g. hydrogen bond and electrostatic). However, a fibre is unattainable to be modeled atomistically due to its large dimensions, and molecular models usually stay at the microfibre scale (Hadden et al. 2013) or even lower (cellulose chains). Usually the MD studies are focused on determining thermodynamics properties such as adsorption (Mazeau and Vergelati 2002; Da Silva Perez et al. 2004; Zhang et al. 2011; Mazeau and Wyszomirski 2012) and adhesion energies (Bergenstråhle et al. 2008), or even structural parameters (Hardy and Sarko 1996; Nishiyama et al. 2008). Wave function studies can also be found on literature, but they are limited to models smaller than in molecular dynamics (Bourassa et al. 2013; Watts et al. 2014). Using steered molecular dynamics (SMD) it is also possible to calculate the force needed to separate two interacting group of atoms (Bergenstråhle et al. 2009), which could be useful to estimate bond properties, and results of these type of simulations showed good agreement with equivalent atomic force microscope (AFM) experiments (Morfill et al. 2008). Nevertheless, few studies have attempted to estimate a value of bond strength from simulation and these are limited to models of two microfibrils (Zhao et al. 2014) or even single cellulose molecules (Bergenstråhle et al. 2009).

It has been shown the importance of understanding how the properties of the fibres, as well as the bonds between the fibres, influence the macroscopic behavior of the final material. Under this consideration the chemical modification of fibre surface is usually carried out to study its effect on the bonds between fibres (having the proper method correctly optimized) and later overall properties (Torgnysdotter and Wågberg 2004), given that the surface chemistry of fibre wall determine the properties of the bonds between the fibres. Chemical modification of fibre cell wall could be carried out in two ways: covalently by the grafting of chemical groups onto the cellulose chains (TrejoO'reilly et al. 1997; Heinze and Liebert 2001); or noncovalently by the adsorption of molecules onto the surfaces of the fibres (Kargl et al. 2012). The use of dry strength additives is a constant in paper industry to produce stronger fibre-fibre joints (Eriksson 2006), where starches, acryl amide-based polymers, gums, or other substances are usually employed to improve the mechanical properties of paper. Amongst all these additives, polyelectrolytes are commonly used as aidretention of fines and fillers (Su et al. 2015), and to increase the strength of the final material (Enarsson 2006; Gimåker 2007). The mechanism of adsorption of polyelectrolytes onto cellulosic fibres is usually described as a pure ion exchange in the literature, where an electrostatic interaction between the charges on the fibres and the charges on the polyelectrolytes is established (Wagberg 2000), although other specific non-electrostatic interactions also coexist during the adsorption (Van de Steeg et al. 1992). Using experimental characterization Eriksson (2006) concluded that the tensile strength of paper sheets modified with polyelectrolyte multilayer were related both with the degree of contact between fibres and with the number of efficient fibre-fibre joints. In addition, this author also found that the amount of adsorbed polyelectrolyte was not the only factor contributing to in-plane paper strength properties, but the structure of adsorbed polyelectrolyte was also important. In this regard, MD simulations or even quantum methods could provide a powerful opportunity to study the modes of interaction with cell wall components, or even to model the adsorption behavior of molecules at cellulose interfaces, complementing experimental measurements (Kargl et al. 2012). Bourassa et al. (2013) studied either the hydration and interaction energies of cellulose nanocrystal (CNC) surfaces without modification or after carboxyl of sulphate modification and observed changes either in the adsorption energy of water and interaction energy between surfaces after modification.

The modeling of a network of fibres from its constituent fibres, taking into account the inherent properties of fibres and bonds between fibres, become thus a challenging goal since the interactions between fibres are produced between several structural dimensions (Lindström et al. 2005). Some authors described the architecture of the inter-fibre joint as a combination of fibre-to-fibre, fibre-to fibril and fibril-to-fibril 
contacts (Van den Akker 1959). Considering in this sense the importance and the role of the intermolecular forces in the inter-fibre joint strength, in the present study we have developed a molecular model of cellulose fibril surfaces (CFS) that allows for the determination of forces and modes of interactions by means of MD simulations. In face of the bibliography related to the ultrastructure of cellulose fibres at the nanometer scale (Ding et al. 2014), we consider that this model could be representative of the interaction between two hypothetical fibres at molecular level. We aim to assess its representativeness and sensitivity as an hypothetical point of contact between two fibres, so the developed CFS models were studied either in the pristine state or after non-covalent surface modification with two types of polyelectrolytes, carboxymethyl cellulose (CMC-ONa) and a cationic polyacrylamide (CPAM).

\section{Methods}

Computational details

All calculations presented in the next study were performed using the LAMMPS simulation code (Plimpton 1995). To model interactions between atoms in the cellulose fibrils and polyelectrolyte molecules throughout the calculation, a Class I potential was employed according to the next mathematical formulation:

$$
\begin{aligned}
\mathrm{E}= & \sum_{\text {bonds }} \mathrm{k}_{\mathrm{r}}\left(\mathrm{r}-\mathrm{r}_{0}\right)^{2}+\sum_{\text {angles }} \mathrm{k}_{\Theta}\left(\Theta-\Theta_{0}\right)^{2} \\
& +\sum_{\text {dihedrals }} \frac{\mathrm{v}_{\mathrm{n}}}{2}[1+\cos (\mathrm{n} \phi-\gamma)]+ \\
& +\sum_{\mathrm{i}<\mathrm{j}}\left[\left(4 \varepsilon_{\mathrm{ij}}\left(\frac{\sigma_{\mathrm{ij}}}{\mathrm{r}_{\mathrm{ij}}}\right)^{12}-\left(\frac{\sigma_{\mathrm{ij}}}{r_{\mathrm{ij}}}\right)^{6}\right)+\frac{\mathrm{q}_{\mathrm{i}} \mathrm{q}_{\mathrm{j}}}{\mathrm{r}_{\mathrm{ij}}}\right. \\
& \left.+\mathrm{D}_{\mathrm{ij}}\left(5\left(\frac{\mathrm{R}_{\mathrm{ij}}}{\mathrm{r}_{\mathrm{ij}}}\right)^{12}-6\left(\frac{\mathrm{R}_{\mathrm{ij}}}{\mathrm{r}_{\mathrm{ij}}}\right)^{10}\right) \cos ^{4} \Theta_{\mathrm{ij}}\right]
\end{aligned}
$$

where $\mathrm{r}_{0}$ and $\Theta_{0}$ represent the equilibrium bond length and angle, respectively, while $\mathrm{k}_{\mathrm{r}}, \mathrm{k}_{\Theta}$ and $\mathrm{v}_{\mathrm{n}}$ are force constants. $\mathrm{n}$ is the multiplicity and $\gamma$ the phase angle for the torsional angle potential. In the case of nonbonded interactions, $\varepsilon_{\mathrm{ij}}$ and $\sigma_{\mathrm{ij}}$ represents respectively the well depth and the collision diameter of the Lennard-Jones potential for the van der Waals interactions, while $\mathrm{q}$ is the partial atomic charge for the Coulombic potential. The hydrogen bonds were modeled explicitly by a $12-10$ potential, where $\mathrm{D}_{\mathrm{ij}}$ and $\mathrm{R}_{\mathrm{ij}}$ are the well depth and distance between the donor and the hydrogen acceptor, respectively, whereas $\Theta_{\mathrm{ij}}$ is the angle formed between the hydrogen donor, the hydrogen (i) and the hydrogen acceptor (j). The value of these parameters were obtained from the Dreiding force field (Mayo et al. 1990). Although this force field doesn't consider some specific characteristic of carbohydrates, such as the anomeric effect (Foley et al. 2012), the Dreiding force field has been employed in the modeling of carbohydrate molecular systems to study interfacial phenomena such as adsorption with good performance (Mazeau and Wyszomirski 2012). On the other hand the interactions between atoms in water molecules were modeled using the TIP3P potential (Jorgensen et al. 1983), allowing for flexibility of bond and angles (no constraints in the molecules). The form of this potential is shown next:

$$
\begin{aligned}
E= & \sum_{\text {bonds }} \mathrm{k}_{\mathrm{r}}\left(\mathrm{r}-\mathrm{r}_{0}\right)^{2}+\sum_{\text {angles }} \mathrm{k}_{\Theta}\left(\Theta-\Theta_{0}\right)^{2} \\
& +\sum_{\mathrm{i}<\mathrm{j}}\left[\left(4 \varepsilon_{\mathrm{ij}}\left(\frac{\sigma_{\mathrm{ij}}}{\mathrm{r}_{\mathrm{ij}}}\right)^{12}-\left(\frac{\sigma_{\mathrm{ij}}}{\mathrm{r}_{\mathrm{ij}}}\right)^{6}\right)+\frac{\mathrm{q}_{\mathrm{i}} \mathrm{q}_{\mathrm{j}}}{\mathrm{r}_{\mathrm{ij}}}\right]
\end{aligned}
$$

The purpose of introducing some water molecules in the system under study was to consider for the random interactions that could be produced by the presence of humidity in the real systems, but not to study specific interactions with the cellulose or polyelectrolytes molecules. Although in the TIP3P potential the hydrogen bonds are not explicitly defined with a specific potential, in contrast with the Dreiding force field, in the TIP3P potential the contribution of hydrogen bonding is considered as a mean field approach through the van der Waals interaction and the high electrical partial atomic charges that are considered in the hydrogen and oxygen atoms of water molecules $(+0.417$ and -0.834 respectively). Finally, the interaction between atoms in water molecules and atoms in the cellulose fibrils and polyelectrolyte molecules were modeled using the Lennard-Jones and Coulombic potential and considering the geometric means for evaluation of the crossterms as follows: 


$$
\begin{aligned}
& \varepsilon_{\mathrm{ij}}=\sqrt{\varepsilon_{\mathrm{ii}} \varepsilon_{\mathrm{jj}}} \\
& \sigma_{\mathrm{ij}}=\sqrt{\sigma_{\mathrm{ii}} \sigma_{\mathrm{jj}}}
\end{aligned}
$$

In the standard form of force fields, both LennardJones and Coulombic potential are usually truncated at distances higher than a certain cut-off value to reduce the number of possible pair calculations, which means that the force and energy become zero at these distances. Although this approximation introduces a certain error in the calculations, in the case of electrostatic interactions it is usual to couple a long range solver to compensate it. Conventionally, these long range electrostatic interactions are evaluated by the Particle Mesh Ewald (PME) approach. Nevertheless, this method uses the reciprocal space to calculate the long range interactions, which means that periodic simulation boxes are needed. In the present study non periodic setups were modeled, using large simulation boxes with large void spaces, which in the case of application of PME method led to low performances. Instead, if the PME solver was deactivated and the cutoff distance of short range Coulombic interactions was set-up judiciously in order to compensate the absence of the long range solver, higher performances could be obtained. Besides, the use of cutoff methods instead of PME solver could provide better results in some systems (Fadrná et al. 2005). Using a single cellulose microfibril (MFC) as a sample model, several Coulombic cut-off distances were tested and the electrostatic energy of the model was analyzed after reaching the equilibrium plateau in NVT ensemble. The results of the optimization are shown in Fig. 1. Considering the model with long range (PME)

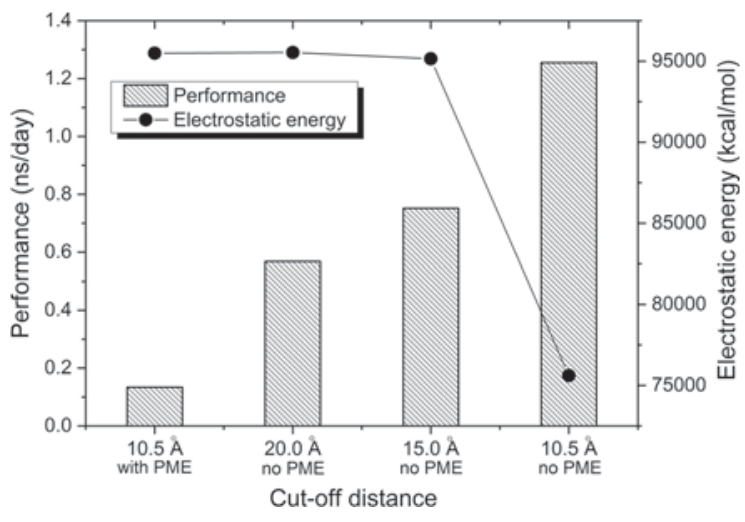

Fig. 1 Adjustment of Coulombic cut-off distance and performance solver and $10.5 \AA$ cut-off distance as reference of energy, it can be observed that if the cut-off distance was maintained at $10.5 \AA$ but the PME solver was switched off, the electrostatic energy decreased dramatically introducing an unacceptable error in the calculations. By increasing then the cut-off distance to 15.0 or $20.0 \AA$, then the electrostatic energy became practically the same value as the reference model (with a difference of less than $1 \%$ in both cases). Since the performance was higher with a cut-off of $15.0 \AA$ than with a cut-off of $20.0 \AA$, with similar results in energy, a cut-off of $15.0 \AA$ was chosen for the Coulombic potential. In the case of Lennard-Jones potential, which was not affected by the PME solver, a standard $10.5 \AA$ cut-off was used as stated in the Dreiding force field. All simulations were performed in vacuo using NVT ensembles to obtain the different atomic configurations, employing the Nosé-Hoover thermostat (Nosé 1984; Hoover 1985) to maintain the systems at $300 \mathrm{~K}$ of temperature. The time step was set at $1.0 \mathrm{fs}$ and the Verlet algorithm was used to update positions of atoms.

\section{System preparation}

Initially, oligomers of cellulose were built by bonding 20 units of $\beta$-D-glucopyranose by $\mathrm{O}-\beta(1 \rightarrow 4)$-glycosidic bonds. Partial atomic charges were taken from Miyamoto et al. (2009) as indicated in Fig. 2a. Considering the crystallographic structure of cellulose microfibrils (MFC) reported in the literature (Nishiyama et al. 2008), a total of 36 of these cellulose oligomers were subsequently placed in the crystallographic points, in an arrangement of 8 layers, to generate a model of MFC. This arrangement is based on the hypothetical rosette structure proposed in the literature (Doblin et al. 2002). The system was placed in the center of a simulation box and was allowed to equilibrate under NVT ensemble during 1 ns to reach a plateau in energy. The final dimensions of this model of cellulose microfibril were about $11.1 \mathrm{~nm}$ length, $5 \mathrm{~nm}$ width and $3 \mathrm{~nm}$ height. Next in the cell wall structure of fibres from one to several microfibrils arranges into bundles to make up a cellulose fibril (O’Sullivan 1997; Wathén 2006), the next level of organization of cellulose in fibres. According to atomic force microscopy (AFM) (Ding et al. 2014) and scanning electron microscopy (SEM) (Zhang et al. 2016) images these fibrils conform a fibrillar layered 
Fig. 2 Partial atomic charges of a neat cellulose, b $\mathrm{CMC}-\mathrm{ONa}$ and c $\mathrm{CPAM}$

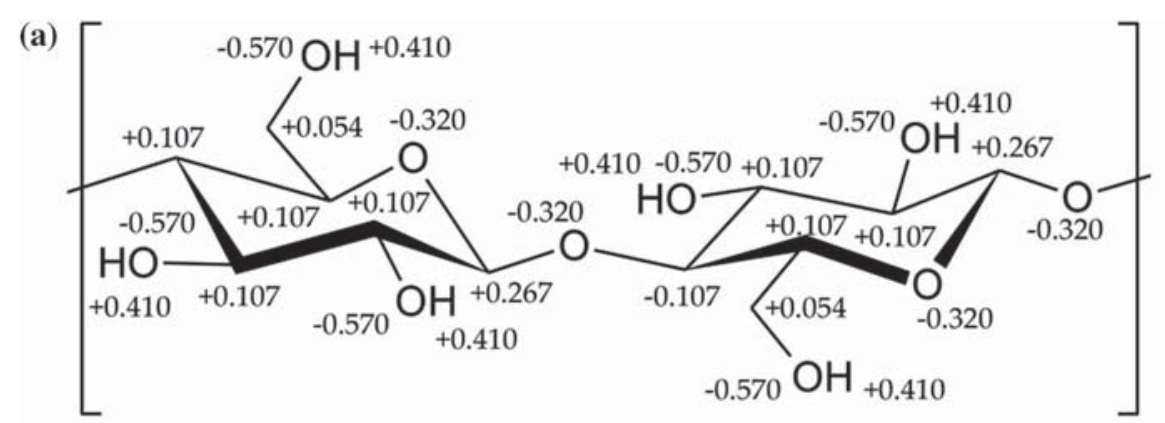

(b)

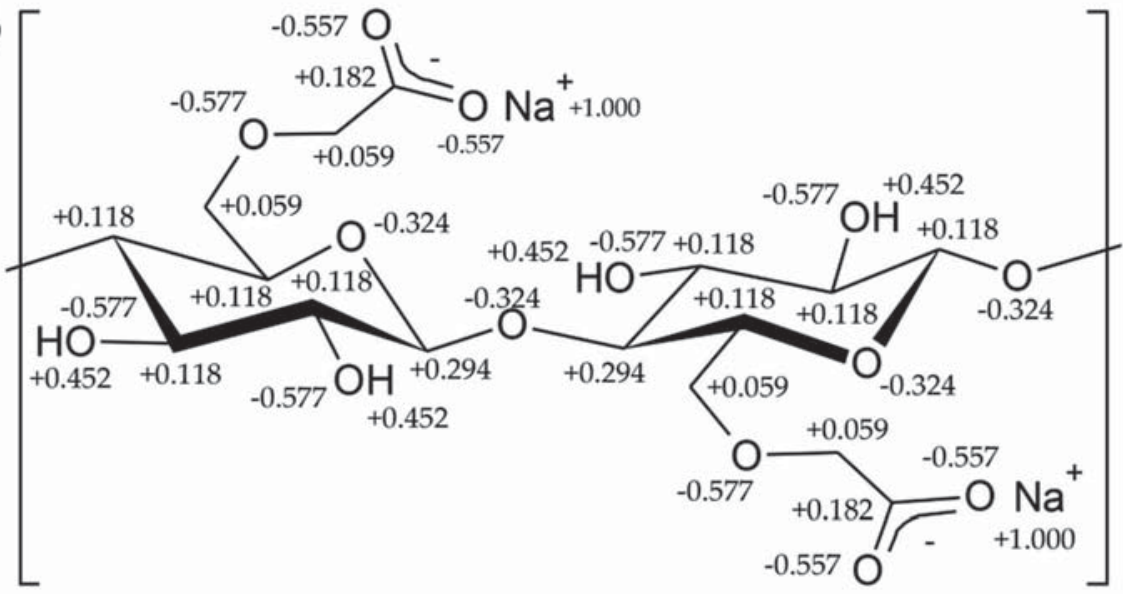

(c)

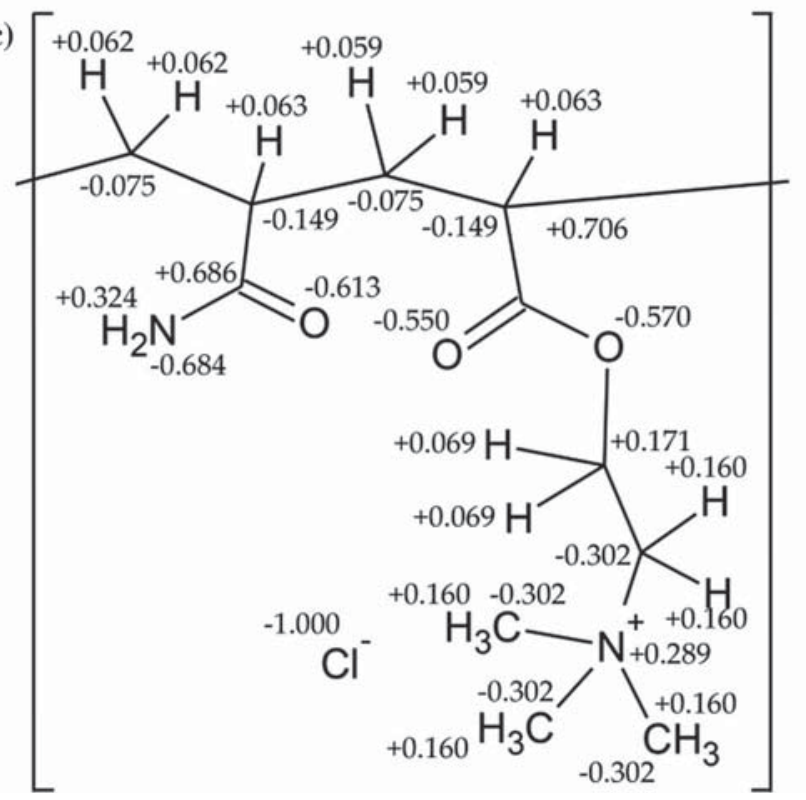

network in which MFCs interact each other through their hydrophilic sides ((110) or (1-10) crystallographic planes), where cellulose chains mainly orient their hydroxyl groups and where the possibility of hydrogen bonding is higher (O'Sullivan 1997). This

type of aggregation of microfibrils have also been observed by molecular dynamics simulations (Oehme et al. 2015). The hydrophobic sides of MFCs ((100) crystallographic planes) are then mainly oriented outwards and compose thus the majority of the 
exposed surface of the fibrils and, consequently, of the fibre (Mazeau 2011). Considering then this organization of MFC into fibrils observed by microscopy, molecular models of cellulose fibril surfaces (here identified as CFS) were constructed by disposing 3 MFCs throughout their hydrophilic sides, exposing thus the hydrophobic sides outwards the fibre, and allowing them to reach energy equilibrium in NVT ensemble during $50 \mathrm{ps}$. A scheme of the modeling procedure of CFS from MFC is shown in Fig. 3. We decided to use this setup of 3 MFCs since it was large enough to accommodate several polyelectrolyte molecules in its surface but without a very demanding computational cost (the developed CFS surface account for a total of 45684 atoms). In this study no ionic groups were included in the molecules of cellulose, so the studied modes of interactions of polyelectrolytes towards the created cellulose fibril surfaces were only produced by a non-electrosorption process (no ion exchange process), although the adsorption of polyelectrolytes onto cellulose fibres is usually associated to a combination of non-electrostatic and a pure ion exchange process (Budd and Herrington 1989; Wagberg 2000). The raw data file generated for a CFS surface, in LAMMPS format, is supplied as Online Resource 1.

Polyelectrolyte molecules were generated by a replication procedure repeating the monomer structure until getting the desired degree of polymerization. In carboxymethyl cellulose (CMC) the ionicity of the molecule is obtained by dissociation of the hydrogen from the carboxylic groups $(-\mathrm{COOH})$ and thus the number of carboxylic groups in the molecule would affect its surface charge. The number of carboxylic groups in CMC derivatives is usually identified as the degree of substitution (DS), which is the number of hydroxyl groups which have been substituted or grafted with carboxymethyl groups $\left(-\mathrm{CH}_{2} \mathrm{COOH}\right)$. In this study a DS of 1.0 was chosen, which meant that all primary hydroxyl groups in glucose residues were grafted with carboxymethyl groups. Since dissociation of carboxylic groups induces a negative charge on these groups, a positive counterion needed to be added to each group to neutralize it. In this way, sodium was chosen as the counterion of anionic CMC molecules, which is usually the most common existing form of CMC (Li et al. 2017). Chains of 50 glycoside residues were built with a molecular weight of $5153 \mathrm{Da}$, each one carrying a $-\mathrm{CH}_{2} \mathrm{COONa}$ ionizable group, and these polyelectrolyte molecules were identified as CMC-ONa. The partial charges of atoms in CMCONa monomers are indicated in Fig. 2b. The structure of cationic polyacrylamide derivatives (CPAM) is obtained from combining two types of monomer units: acrylamide (AM) and methacryloyloxyethyl trimethyl ammonium chloride (TMAEMC). In this type of polymers the ionicity is determined by the number of ammonium monomers (TMAEMC) over the total of monomers present in the chain. Chains of a total of 50 monomers were built using 44 monomers of AM and 6 monomers of TMAEMC, thus making a $12 \%$ cationicity of CPAM molecule models and a molecular weight of $4291 \mathrm{Da}$. TMAEMC were homogeneously distributed in the chain, with 7 non-cationic monomers of AM in between each ammonium monomer. With respect of the tacticity, all the ammonium and amide groups were placed at the same side of the chain and thus the isotactic variety of the polymer was obtained. Partial atomic charges of CPAM monomers are indicated in Fig. 2c. For each type of polyelectrolyte

Fig. 3 Modeling scheme of CFS (labeling of surfaces according to Mazeau (2011))

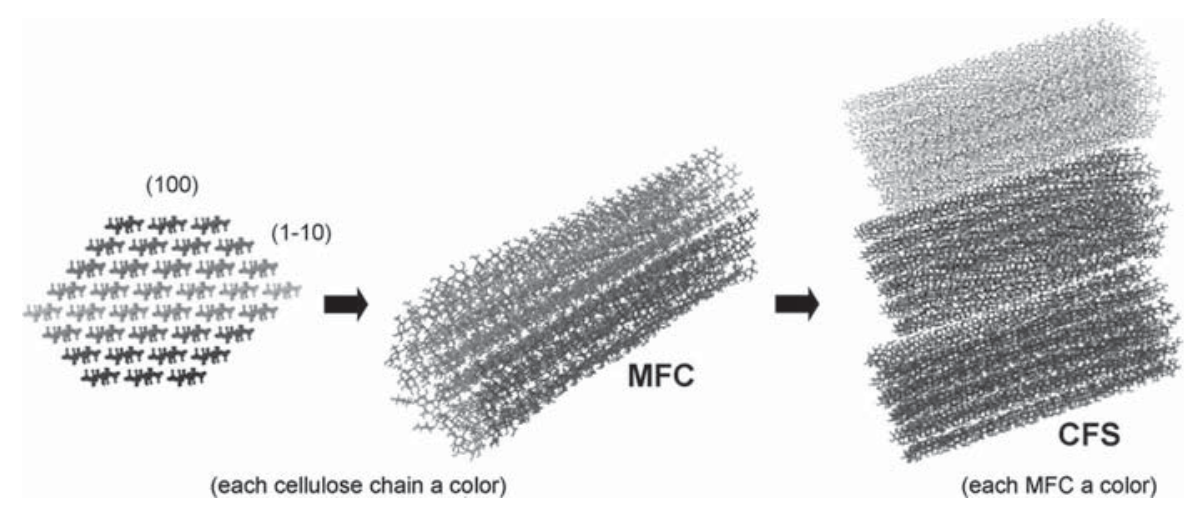


the raw data files generated for the single molecule structures, in LAMMPS format, are supplied as Online Resources 2 and 3.

The CFS joint models developed in the present study are considered as representative points of attachment or contact between fibrils in cell walls. To generate these models, two of the CFS models were first placed within a simulation box of cubic dimensions ( $220 \AA$ side). In Fig. 4 a graphic representation of the coupling scheme is depicted. Initially the CFS were placed at a distance of about $6 \AA$ and a relative angle orientation of near $45^{\circ}$ between them. In the case of modified CFS the initial distance was higher (30 ̊) to allow for the accommodation of the required polyelectrolyte molecules (from 1 to 3 ) between the CFS. The initial position of the polyelectrolyte molecule/s respect to the fibril surface was placed in the region between two consecutive MFC in the fibril bundle towards the (1-10) crystallographic plane. Note that the structure of polyelectrolyte molecules was previously equilibrated in isolated vacuum simulations during 10 ps in NVT ensemble. Explicit water molecules were also randomly dispersed into the simulation box considering moisture of $6.5 \%$ in weight to better reproduce the conditions of the real materials (this is usually the moisture of cellulose materials at $300 \mathrm{~K}$ and $50 \%$ of relative ambient humidity according to Levlin and Söderberg (1999)). Reflective walls were applied to the simulation box in order to assure the complete adsorption of water molecules on the CFS. To bring the two CFS closer, a smooth force of $150 \mathrm{nN}$ was initially applied during $10 \mathrm{ps}$ in NVT ensemble over one of the CFS perpendicular to its surface and oriented towards the other CFS. After this time the force was set to zero and the models were let to equilibrate under NVT ensemble during a total time of $1 \mathrm{~ns}$. In this final step the time integration was applied by groups to avoid rejection of molecules or undesired desorption of polyelectrolyte molecules due to repulsion forces. The first $100 \mathrm{ps}$ were only applied to the lower CFS, maintaining the polyelectrolyte molecule/s and the upper CFS frozen. Then another 100 ps were only applied to the upper CFS and other 100 ps were applied again to the lower CFS. After that, either the upper CFS or the polyelectrolyte molecule/s were time integrated during another 100 ps to finally allow the whole system to equilibrate in additional 600 ps (thus making a total integration time of $1 \mathrm{~ns}$ ) to reach the equilibrium. Water molecules were always time integrated. We checked that the applied integration time of $1 \mathrm{~ns}$ allowed to reach a plateau in the potential energy curves, which allowed to get stable geometries and pair correlation functions $(g(r))$ of the systems under study. Besides all water molecules were adsorbed into the CFS after this integration time. In Fig. 5 a representation of the each modified CFS model is shown, in which one of the CFS has been omitted. It can be seen from this representations that the structure of adsorbed polyelectrolytes was similar to a deformed globule, which is a typical conformation for hydrophobic polyelectrolytes over solid substrates (Minko et al. 2002). Note also that although the fibril surfaces interacted mainly through the (100) faces in the developed models, the polyelectrolytes mainly adsorbed in the (1-10) face

Fig. 4 Coupling scheme of CFS and polyelectrolyte molecules into the final setup

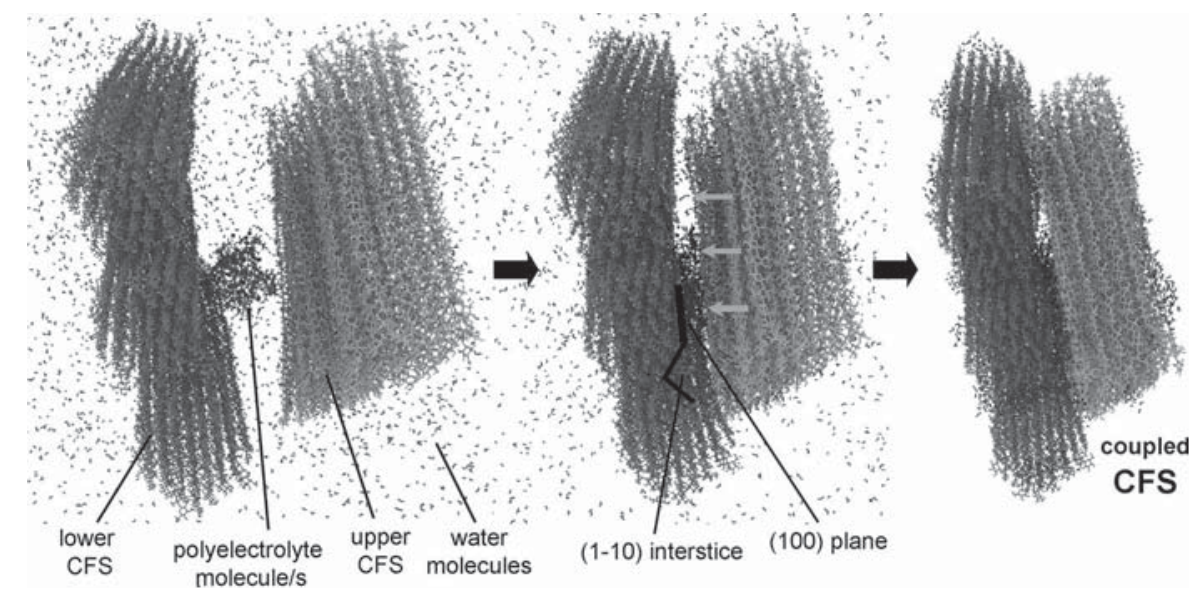


Fig. 5 Molecular models of modified CFS (upper fibril is omitted from

representations and polyelectrolyte molecules are pointed out by arrows)
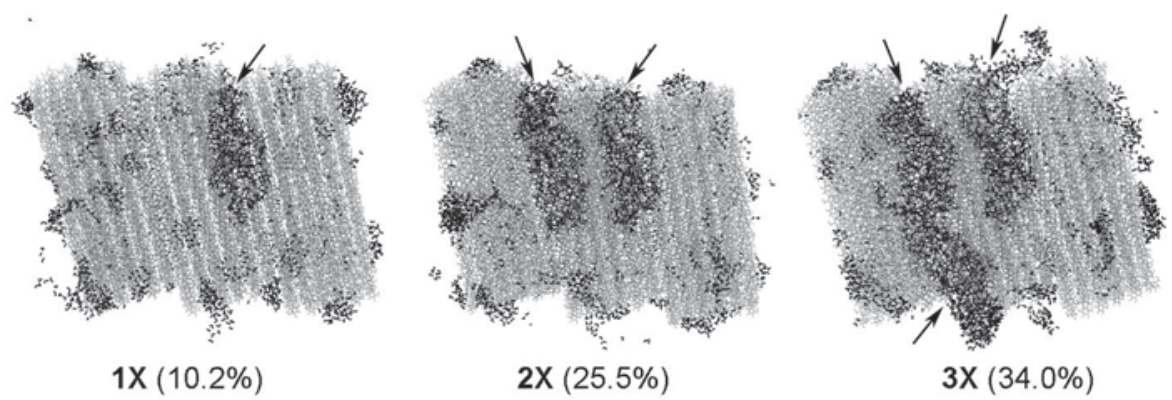

CMC-ONa surface concentration
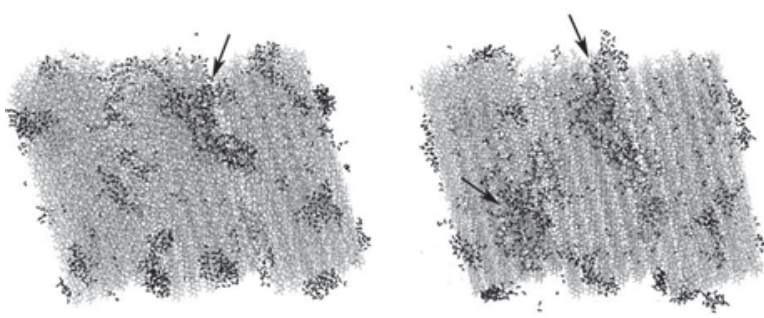

$2 X(20.3 \%)$

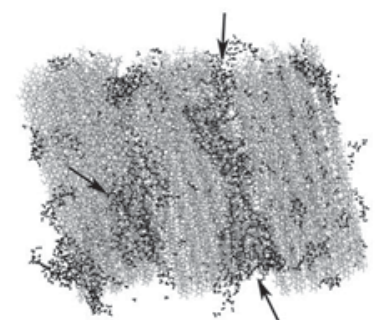

$3 X(23.1 \%)$

\section{CPAM surface concentration}

interstices which were located between consecutive bonded microfibrils.

\section{Assessment of polyelectrolyte interactions}

The radial distribution functions (RDF), also called pair correlation function $\mathrm{g}(\mathrm{r})$, were obtained to study specific interactions between polyelectrolytes and cellulose fibrils. These functions were obtained using the corresponding compute command of LAMMPS code and selecting only non-bonded interactions (intramolecular interactions were dismissed from the analysis) between selected atom pairs of the ionic groups of the polyelectrolyte molecules (sodium carboxylate in $\mathrm{CMC}-\mathrm{ONa}$ and chloride ammonium groups in CPAM) and all atoms in cellulose fibrils. Bins of $0.075 \AA$ size $(\Delta r)$ were defined for the sorting procedure to calculate the RDF functions, which were finally obtained by averaging profiles obtained over a period of $10 \mathrm{ps}$ at intervals of $0.1 \mathrm{ps}$ each one. RDF functions $(\mathrm{g}(\mathrm{r}))$ were calculated according to next formula:

$g_{i j}(r)=\frac{n_{i j}(r)}{4 \pi r^{2}}$ where $n_{i j}(r)$ is the number of pair atoms $i-j$ within the range $((r-\Delta r / 2, \quad r+\Delta r / 2)$. Each function was normalized by dividing with the total area under the curve. After the equilibration of the models in the NVT ensemble during $1 \mathrm{~ns}, \mathrm{~g}(\mathrm{r})$ was sampled at intervals of 0.1 ps during 10 ps to calculate an average pair correlation function.

In addition to RDF the efficiency of bonding between fibrils was calculated as the relative bonded area $\left(\mathrm{RBA}_{\mathrm{m}}\right)$ between cellulose fibrils. In this case the relative bonded area is defined as the fraction of surface, from the total exposed to the bonding, which is in intimate contact between fibrils. To calculate the $\mathrm{RBA}_{\mathrm{m}}$ the next formula was used:

$\mathrm{RBA}_{\mathrm{m}}=\frac{\mathrm{A}_{\text {contact }}}{\mathrm{A}_{\text {total }}}$

where $A_{\text {total }}$ is the total area and $A_{\text {contact }}$ is the area in contact between cellulose fibrils. The total area $\left(\mathrm{A}_{\text {total }}\right)$ was calculated as the common projected area between cellulose fibrils (see an illustrative picture in Fig. 6), whereas the area in contact $\left(\mathrm{A}_{\text {contact }}\right)$ was obtained using "surface mesh" utility from the OVITO software (Stukowski 2010) and setting up a probe atom radius of $3.5 \AA$ and a smoothing level of 100 . Since this relative bonded area only refers to the fraction of 


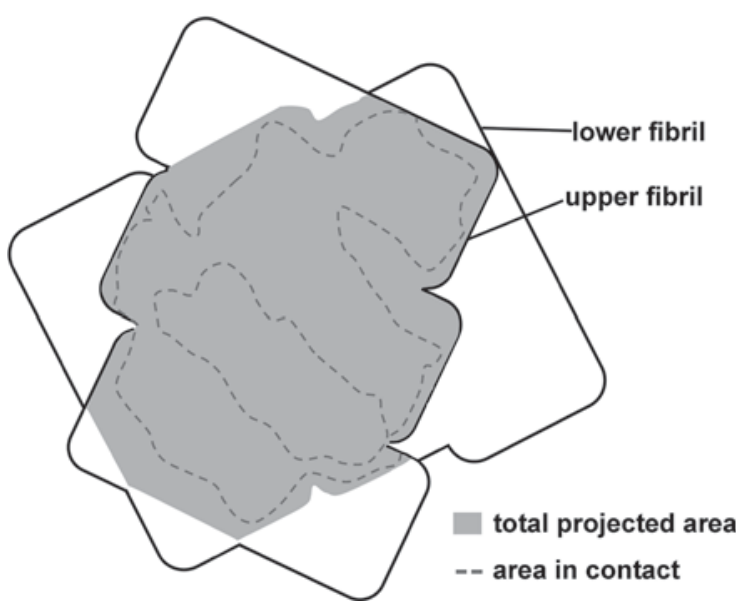

Fig. 6 Calculation of $\mathrm{RBA}_{\mathrm{m}}$ from projected and area in contact

surface which is in direct molecular contact from the total surface exposed between fibrils, the " $\mathrm{m}$ " subscript was used to distinguish it from the macroscopic RBA (Batchelor and Jihong 2005).

Pull out simulations (SMD)

The force of adhesion existing in between CFS was calculated through pull out simulations. After equilibration of the CFS models, neat or polyelectrolyte modified, non-equilibrium or steered molecular dynamics (NEMD or SMD) was carried out to perform pulling out of one of the fibrils to the other. One of the CFS was fixed by applying external constrains to the cellulose chains which were not in direct contact with the other CFS, letting the rest of chains in contact with the other CFS freely to move during time integration. In this way the flexibility of the contact zone between CFS was not perturbed due to the constraints applied, while allowed to fix one of the two CFS to perform pull out. The other CFS was pulled out from the fixed CFS by applying an external force to all its constituent atoms. This force was applied in a direction perpendicular to the contact plane between CFS and increased linearly with the simulation time according to next formula:

$\mathrm{F}_{\text {pull }}(\mathrm{t})=\mathrm{F}_{0}+\mathrm{j} \cdot \mathrm{t}$

where $F_{0}$ is the force at time zero, $\mathrm{j}$ is the jerk (rate of change of force) and $t$ is the simulation time. In this simulation, $\mathrm{j}$ was fixed at $0.159 \mathrm{nN} \mathrm{\textrm {ps } ^ { - 1 }}$. This pull out procedure would correspond to testing mode I of the joint (tensile opening mode), so the calculated fibril bond strength corresponded to the normal component. The displacement of the pulled out CFS as well as the potential energy was monitored during simulation every $0.1 \mathrm{ps}$ in order to calculate the force at the complete pull out. The strength between fibrils was calculated by normalizing to the total projected area according to next formula:

$\mathrm{S}=\frac{\mathrm{F}_{\text {pull }}}{\mathrm{A}_{\text {total }}}$

where $A_{\text {total }}$ was calculated from image analysis. Six replicas of the pull out process were performed over each CFS model and finally averaged to obtain an estimation of the bond strength between fibrils. During the pull out simulations the total number of binding interactions (which come from the sum of van der Waals, Coulombic and hydrogen bond) were also monitored in order to have an estimation of the density of interactions (interactions per unit area) between fibrils. This calculus was made according to next formula:

(density of interactions $)=\frac{\mathrm{n}_{0}-\mathrm{n}_{\mathrm{end}}}{\mathrm{A}_{\text {total }}}$

where $\mathrm{n}_{0}$ is the number of interactions at the beginning of pull out simulation and $n_{e n d}$ is the number of interactions after complete pull out. Finally a "strength factor" was calculated as the product given of the $\mathrm{RBA}_{\mathrm{m}}$ and the total number interactions measured from pull out simulations, as stated in the next formula:

Strength factor $=\mathrm{RBA}_{\mathrm{m}} \cdot($ density of interactions $)$

\section{Results and discussion}

Analysis of interactions between polyelectrolytes and (1-10) cellulose fibril surfaces

After reaching the equilibrium state, the interactions between the two studied polyelectrolytes, i.e. CMC$\mathrm{ONa}$ and CPAM, with cellulose fibril surfaces through the (1-10) hydrophilic surface were studied in terms of the radial distribution function (RDF). The radial distribution function or pair correlation function $g(r)$ is 
a useful way to describe the molecular structure, and gives the probability of finding an atom a distance $r$ from another atom compared to the ideal gas distribution (Leach 2001). In Fig. 7 the RDF for intermolecular interactions between polyelectrolytes molecules and cellulose fibrils are plotted for each type of analyzed polyelectrolyte molecule. These profiles were generated by averaging the curves obtained for each of the analyzed polyelectrolyte surface concentration setups (identified as $1 \mathrm{X}, 2 \mathrm{X}$ and $3 \mathrm{X}$ ). Note also that intramolecular interactions (those from covalent bonds and non-bonded interactions that are produced within a molecule) were not considered in these profiles.

In the case of the $\mathrm{CMC}-\mathrm{ONa}$ polyelectrolyte (Fig. 7a) a sharp peak was identified at $2.1 \AA$, which corresponded also to the maximum intensity for all analyzed surface concentrations. This peak was
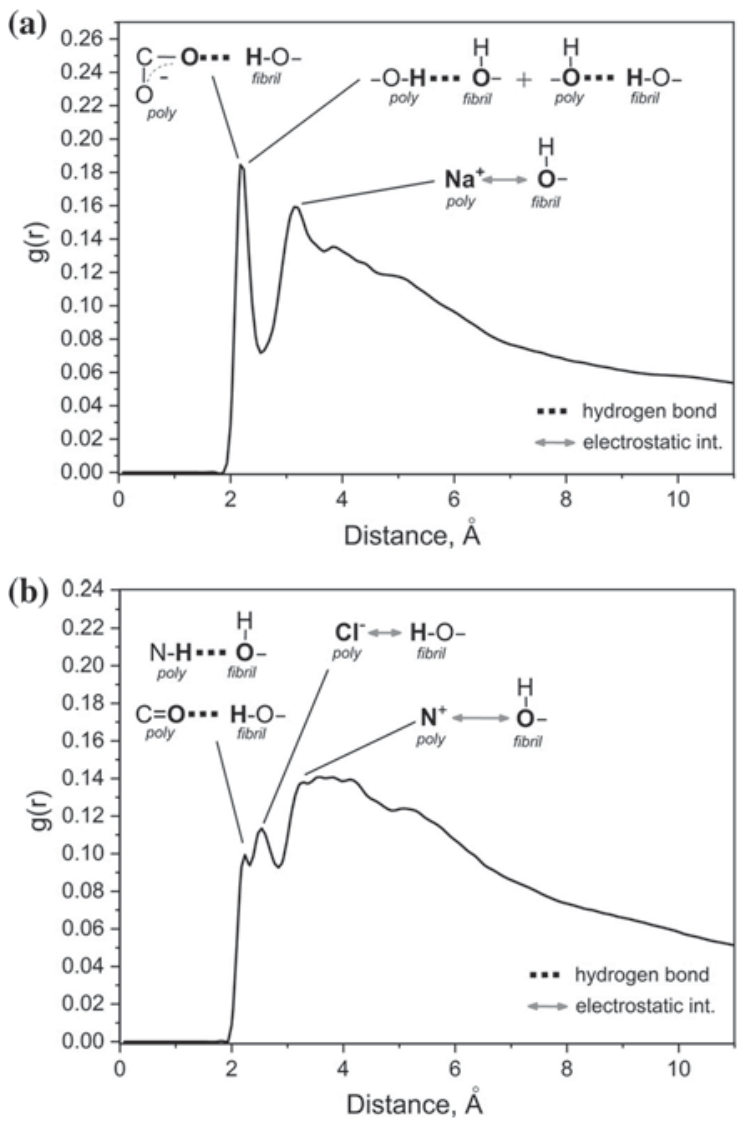

Fig. 7 Averaged radial distribution functions (RDF) of intermolecular interaction between (1-10) hydrophilic cellulose surface with a CMC-ONa and b CPAM associated to the hydrogen bonding between the carboxyl oxygens of $\mathrm{CMC}-\mathrm{ONa}$ molecules and hydroxyl groups $(\mathrm{OH})$ in cellulose, and between two hydroxyl groups in between the $\mathrm{CMC}-\mathrm{ONa}$ and the cellulose molecules indistinctly. At higher distances, a second maximum peak was identified at around 3.1 A, which corresponded to the equilibrium distance of electrostatic interaction between the sodium ions of $\mathrm{CMC}-\mathrm{ONa}$ molecules and partial negatively charged oxygen atoms of hydroxyl groups in cellulose molecules. A graphic representation of these identified interactions between the $\mathrm{CMC}-\mathrm{ONa}$ molecules and the cellulose surface (CFS) are depicted in Fig. 8. The mode of interaction shown in Fig. 8a corresponded to pure hydrogen bonding between hydroxyl groups in polyelectrolyte and cellulose molecules. The second mode of interaction (Fig. 8b) corresponded to a combination of either electrostatic and hydrogen bonding interaction, in which networks of sodium ions (or sodium bridges) and carboxyl groups in the $\mathrm{CMC}-\mathrm{ONa}$ molecules interacted with hydroxyl groups in the cellulose by the cited interactions. In its dissertation thesis, Biermann (2001) conducted similar studies and also encountered the formation of sodium bridges in the adsorption of CMC polyelectrolyte to cellulose surfaces. These calculated modes of interaction between the $\mathrm{CMC}-\mathrm{ONa}$ polyelectrolyte and cellulose have also been observed experimentally in other types of substrates. Wang and Somasundaran (2005) found that infrared (FTIR) bands associated with the $\mathrm{C}-\mathrm{O}$ stretch coupled to the $\mathrm{C}-\mathrm{C}$ stretch and $\mathrm{O}-\mathrm{H}$ deformation experienced significant changes upon adsorption of CMC into talc, which supported the strong hydrogen bonding of CMC to the solid surface. They established that the main driving forces for CMC adsorption on talc were a combination of electrostatic interaction and hydrogen bonding rather than hydrophobic force (van der Waals). Although talc is a mineral structure its surface is also composed of hydroxyl groups as in cellulose, so the proposed mode of adsorption of CMC-ONa molecules to cellulose would be consistent with a hydroxyl containing substrate.

The CPAM molecules exhibited the RDF profiles shown in Fig. 7b. At low distances two peaks were identified at around $2.1 \AA$ and $2.5 \AA$. The first peak was associated hydrogen bond interactions, one associated to the interaction between the carbonyl group of CPAM molecules and hydroxyl groups of cellulose, 
Fig. 8 The observed modes of interaction of $\mathrm{CMC}-\mathrm{ONa}$ with non ionic (1-10) hydrophilic cellulose surface: a pure hydrogen bonding and $\mathbf{b}$ electrostatic coupled with hydrogen bonding showing sodium bridges

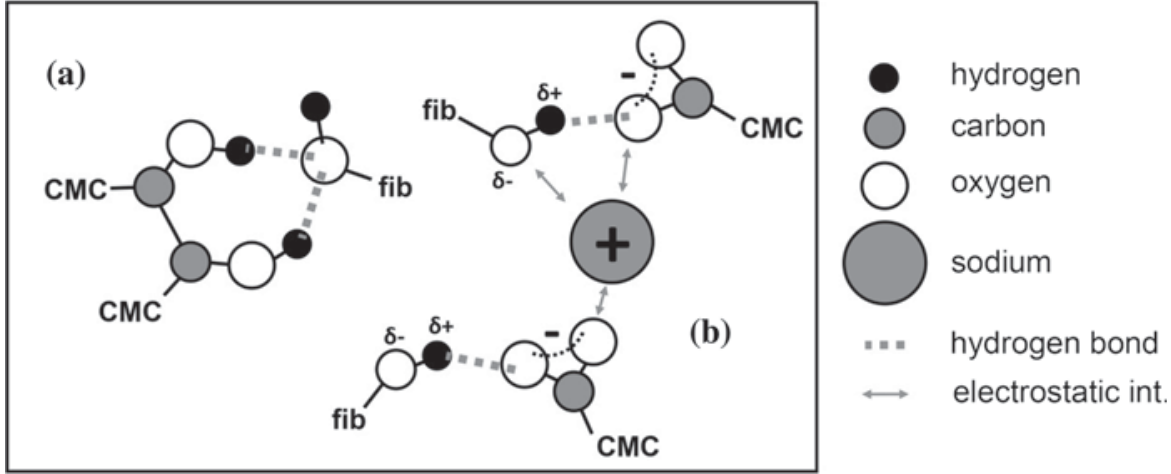

and the other one with the interaction between amine groups in CPAM molecules and hydroxyl groups of cellulose. The second peak, which appeared closely, was associated to the electrostatic interaction between chloride ions and hydrogen atoms of hydroxyl groups in cellulose. Later, at distances higher than $3.0 \AA$, it was observed an increased of RDF intensity which was associated to the electrostatic interaction between the ammonium nitrogen in CPAM and the hydroxylic oxygen in cellulose (at $3.2 \AA$ ) together with other less specific interactions due to van der Waals and proximity of atoms due to other specific interactions. The two modes of interaction between the CPAM polyelectrolytes molecules and the cellulose fibrils proposed in Fig. 9 are based on this RDF. As can be observed, the mode depicted in Fig. 9a corresponds to pure hydrogen bonding and this mode would associate to the amide moieties of the CPAM (to the non-ionic acrylamide monomers). On the other hand, the mode shown in Fig. 9b corresponds to the electrostatic interaction between the ions in the TMAEMC monomers (chloride and ammonium) and the polarized hydroxyl group. From the snapshot visualization it was observed that some of the ammonium groups in the CPAM molecules were totally dissociated by water molecules and chlorine ions were far apart from the ammonium cation. In contrast, in the case of the $\mathrm{CMC}-\mathrm{ONa}$ molecules the sodium cations were tight bonded to the carboxylate groups, due to the smaller size of the implicated ions, and no significative dissociation of the ionic pair was observed. Li et al. (2017) also found the strong interaction of sodium ions with carboxyl groups through MD simulations. The dissociation of the ionic groups would affect in this way the availability of monatomic ions (sodium or chlorine) to generate coupled interactions (as was observed in the $\mathrm{CMC}-\mathrm{ONa}$ ) of the ion pair towards the cellulose.

Experimental studies have shown that the polyelectrolyte adsorption to cellulose substrates is usually associated to pure electrosorption, i.e. an ion exchange process, and non-electrostatic interactions (Wagberg 2000). Here from our results we have shown that the polyelectrolyte molecules can interact with cellulose surfaces with no need of ion exchange (and no release of ions then after the process of adsorption) between each component, which could explain the non-stoichiometric adsorption of polyelectrolytes towards cellulose (Winter et al. 1986). As can be observed from our study, the electrostatic interaction of polyelectrolytes could also be developed with the polarized hydroxyl groups in cellulose.

Evaluation of the bond strength between cellulose fibril surfaces (mode I)

Through pull out simulations of one of the CFS it was possible to measure the strength between fibrils, i.e. the force needed to separate cellulose fibrils. The potential energy was monitored during the pull out simulation (see Fig. 10) and it was observed that due to initial separation of the fibrils the potential energy increased. Before the effective separation of fibrils the potential energy reached a maximum and, just after the separation, the potential energy started to decrease sharply due to the loss of interactions between fibrils. The force at maximum potential energy was considered as the force needed to separate fibrils (rupture force) and this value was used to calculate the strength between fibril surfaces. 


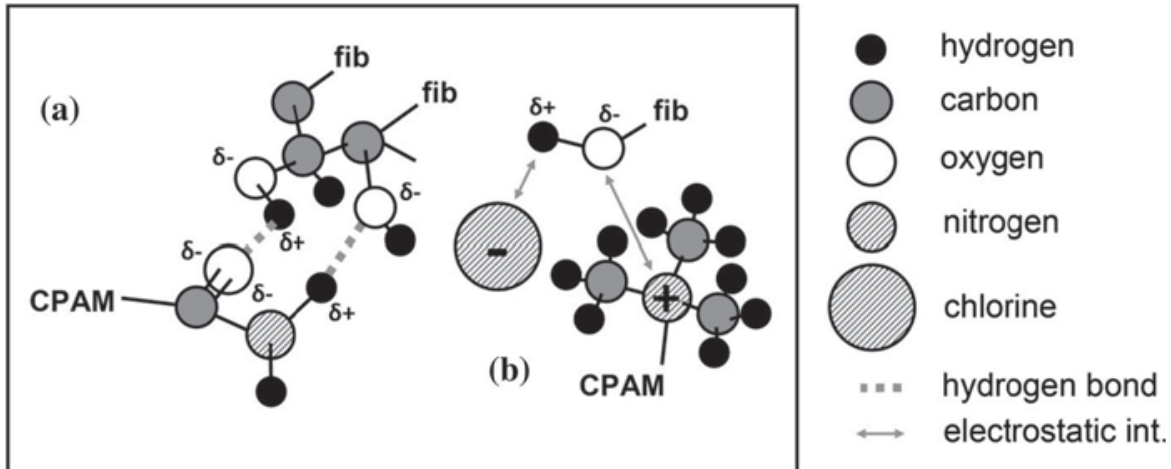

Fig. 9 The observed main modes of interaction of CPAM with (1-10) hydrophilic cellulose surface: a hydrogen bonding and b electrostatic

The results of pull out simulations of the fibrils are summarized in Table 1 in terms of bond strength between fibrils and strength factor, whereas a graphic representation of these results is plotted in Fig. 11. The relative bonded area $\left(\mathrm{RBA}_{\mathrm{m}}\right)$, which is a measure of efficiency of bonding, is also included in the results. Note that these results are referred to normal component or mode I of loading of the bond strength, since the pull out force was applied perpendicular to the contact plane between fibrils. The neat CFS model exhibited an average bond strength of $123.0 \mathrm{MPa}$ and a $\mathrm{RBA}_{\mathrm{m}}$ of 0.651 . This value of bond strength is between two and three orders of magnitude higher than the interfibre joint strength values obtained experimentally at the micrometer fibre scale, which typically lies in the range from 0.2 to $13.9 \mathrm{MPa}$ depending on the type of precedence of the pulp

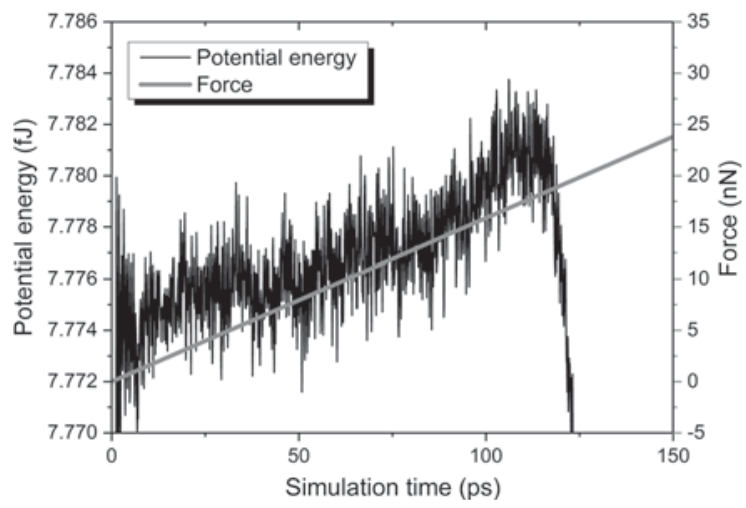

Fig. 10 The evolution in potential energy and force applied versus simulation time during pull out
(Magnusson et al. 2013). Few studies have attempted to calculate the bond strength at lower material scales (fibril or microfibril) from simulation and reported similar values. Zhao et al. (2014) studied the failure stress for an adhesive model of two cellulose microfibrils (CMF) sandwiching an oligosaccharide molecule of xyloglucan through the I $\beta$ (1-00) surface. They obtained a force of around $200 \mathrm{MPa}$ by molecular dynamics simulations, which is near to the values obtained for the CFS models reported in the present study. As they stated, the calculated bond strength indeed represents an upper bound on the true quasistatic value due to the finite pulling speed employed in the simulation. In our simulations the rate of pulling speed during the bonded stage could be approximated as nearly constant and was about $2.5 \mathrm{~nm} \mathrm{~ns}^{-1}$ (the rate of change of force was $0.16 \mathrm{nN} \mathrm{ps}^{-1}$ ). This value of pulling speed was extremely high considering equivalent AFM experiments, which usually gave place to higher values of the barrier force (Morfill et al. 2008). In order to assess the effect of the rate of pulling speed on the properties obtained from pull-out simulations, such as the bond strength or even the strength factor, several rates of change of force (defined as $j$ in Eq. 7) (which are equivalent to different pulling speed rates) were tested on the neat CFS model. The results of this sensitivity analysis are summarized in Table 2 . The rate of change of force had a clear impact on the calculated bond strength and it was observed that the higher the rate of change of force the higher the bond strength between fibril surfaces obtained from pull out simulations. These results were in accordance with the fact that in AFM pulling experiments the binding force 
Table 1 The bonding strength (normal component) between fibrils on (100) hydrophobic surface according to pull out simulations (mode I)

\begin{tabular}{|c|c|c|c|c|c|c|}
\hline \multicolumn{3}{|l|}{ Model } & \multirow{2}{*}{$\begin{array}{l}\text { Bond strength } \\
\text { (MPa) }\end{array}$} & \multirow{2}{*}{$\begin{array}{l}\text { Relative bonded area } \\
\left(\mathrm{RBA}_{\mathrm{m}}\right)\end{array}$} & \multirow{2}{*}{$\begin{array}{l}\text { Density of } \\
\text { interactions, }\left(\AA^{-2}\right)\end{array}$} & \multirow{2}{*}{$\begin{array}{l}\text { Strength factor }{ }^{\mathrm{a}} \\
\left(\AA^{-2}\right)\end{array}$} \\
\hline Surfactant & $\begin{array}{l}\text { Surfactant } \\
\text { molecules }\end{array}$ & $\begin{array}{l}\text { Surfactant } \\
\text { concentration }(\%)\end{array}$ & & & & \\
\hline Neat CFS & - & - & $123.0 \pm 3.0$ & $0.651 \pm 0.014$ & $217 \pm 4$ & $141 \pm 5$ \\
\hline \multirow{3}{*}{$\begin{array}{c}\mathrm{CMC}- \\
\mathrm{ONa}\end{array}$} & $1 \mathrm{X}$ & 10.2 & $118.0 \pm 3.5$ & $0.503 \pm 0.018$ & $187 \pm 8$ & $94 \pm 6$ \\
\hline & $2 X$ & 25.5 & $145.9 \pm 5.0$ & $0.694 \pm 0.015$ & $299 \pm 14$ & $208 \pm 11$ \\
\hline & $3 X$ & 34.0 & $160.8 \pm 6.8$ & $0.711 \pm 0.011$ & $296 \pm 25$ & $211 \pm 20$ \\
\hline \multirow[t]{3}{*}{ CPAM } & $1 \mathrm{X}$ & 6.5 & $126.3 \pm 4.5$ & $0.643 \pm 0.016$ & $267 \pm 18$ & $173 \pm 12$ \\
\hline & $2 \mathrm{X}$ & 20.3 & $143.7 \pm 6.2$ & $0.782 \pm 0.009$ & $283 \pm 23$ & $221 \pm 19$ \\
\hline & $3 \mathrm{X}$ & 23.1 & $137.6 \pm 8.4$ & $0.770 \pm 0.003$ & $287 \pm 32$ & $221 \pm 25$ \\
\hline
\end{tabular}

The first three columns of the table indicate the model in terms of the surfactant, the number of surfactant molecules and the surface concentration of surfactant. Next to these columns are collected the properties of these models in terms of bond strength (in MPa), the relative bonded area (molecular), the density of interactions (in $\AA^{-2}$ ) and the strength factor (in $\AA^{-2}$ )

${ }^{\mathrm{a}}$ Strength factor $=\mathrm{RBA}_{\mathrm{m}} \cdot($ density of interactions $)$
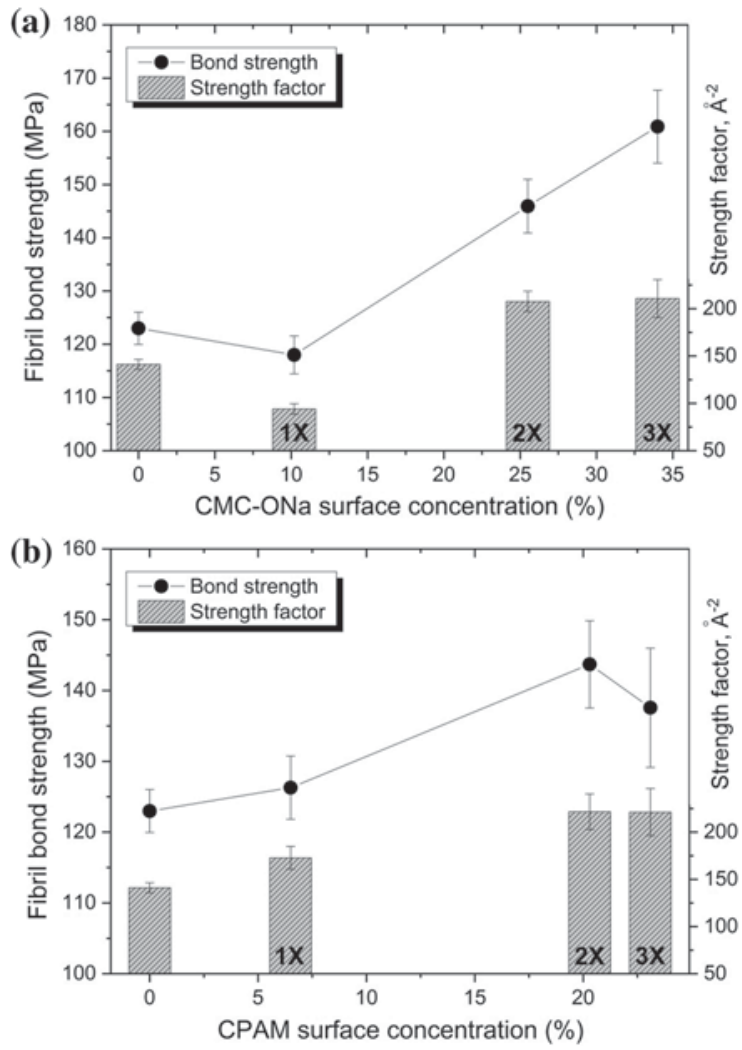

Fig. 11 Fibril bond strength and strength factor versus surface concentration for a CMC-ONa and $\mathbf{b} \mathrm{CPAM}$ is also dependent on pulling speed (Ikai et al. 2007), and were coherent with a viscoelastic response of the inter-fibre joint (Schmied et al. 2013). In the case of the strength factor, which depend on the $\mathrm{RBA}_{\mathrm{m}}$ and the total number of binding interactions per unit area, the effect of rate of change of force was less significative than in the bond strength and the variations obtained were in the range of the uncertainty of the own property (see Table 1). The fact that the rate of pulling speed affected the calculated value of bond strength implies that the pulling parameters (value of $\mathrm{F}_{0}$ and $\mathrm{j}$ in Eq. 7) must be kept constant in order to obtain comparable results. As a final consideration it should be observed that lower pulling speed values also implies higher computational times to obtain the rupture or break point until the unbinding of fibrils occurs, but results tend to be more comparable to real conditions in AFM experiments or other equivalent techniques. Given that the purpose of the present study was to obtain comparable results and to assess the sensitivity of the purposed CFS models to different binding conditions, the value of $\mathrm{j}$ was fixed at $0.159 \mathrm{nN} \mathrm{ps}^{-1}$.

Lindström et al. (2005) stated that in cellulose fibre materials the strength of the inter-fibre joint (or the energy needed to completely separate the joined fibres) is directly proportional to the product of the specific bond strength and the relative bonded area, i.e. the strength between fibres is the result of the intensity 
Table 2 Sensitivity analysis of the effect of rate of pulling speed on the bond strength

\begin{tabular}{lccc}
\hline Rate of change of force $\left(\mathrm{nN} \mathrm{ps}^{-1}\right)$ & Bond strength $(\mathrm{MPa})$ & Strength factor $\left(\AA^{-2}\right)$ & Total simulation time to break point $(\mathrm{h})$ \\
\hline 0.00795 & 79.8 & 154 & 4274 \\
0.0795 & 96.6 & 141 & 480 \\
0.159 & 123.0 & 141 & 314 \\
0.318 & 155.4 & 138 & 186 \\
0.477 & 176.0 & 131 & 134 \\
\hline
\end{tabular}

The first column indicates the value of rate of change of force or jerk (in $\mathrm{nN} \mathrm{ps}^{-1}$ ) and the next columns the resulted bond strength (in $\mathrm{MPa}$ ), the strength factor (in $\AA^{-2}$ ) and the total simulation time required to break point (in hours)

of interactions (reflected in bond strength) and the fraction of surface in contact (assessed by RBA). Under this consideration, the cellulose fibril surfaces (CFS) models presented here were non-covalently modified with polyelectrolyte molecules in order to affect these bond parameters and thus analyze its effect on the resulting bond strength values, as can be seen in Table 1 and Fig. 11. In the present study a strength factor was defined as the product of $\mathrm{RBA}_{\mathrm{m}}$ and density of interactions (as an estimator of specific bond strength) in order to make this assessment. In general lines it was observed a good correlation between the CFS bond strength and the associated strength factor after polyelectrolyte modification or, in other words, it was seen that the bond strength was directly proportional to the product of relative bonded area and total number (density) of binding interactions. At this point it should be noted that the modification with polyelectrolytes could produce either the formation of new interactions or breaking of some of the previous existing interactions between fibrils, so that the density of interactions between fibrils, together with the $\mathrm{RBA}_{\mathrm{m}}$, could either increase or decrease after the modification with polyelectrolytes. Taking first the $\mathrm{CMC}-\mathrm{ONa}$ models (see Fig. 11a), at $1 \mathrm{X}$ surface concentration it was observed a decrease in the bond strength in comparison with the neat CFS model which was due to a reduction both in the relative bonded area $\left(\mathrm{RBA}_{\mathrm{m}}\right)$ and density of interactions between the between the fibril surfaces due to the presence of the polyelectrolyte molecule. When adding more polyelectrolyte molecules between the cellulose fibril surfaces (here depicted as $2 \mathrm{X}$ and $3 \mathrm{X}$ surface concentrations) it was produced an increase on the strength factor, due to an increase either in $\mathrm{RBA}_{\mathrm{m}}$ or density of interactions, in the CFS which finally resulted in higher bond strength values compared to neat CFS. Although both the $2 \mathrm{X}$ and $3 \mathrm{X}$ model had a similar strength factor, the $3 \mathrm{X}$ model exhibited higher bond strength, which indicated that other parameters not considered in the strength factor, in addition to $\mathrm{RBA}_{\mathrm{m}}$ and total number of interactions, also contributed to the bond strength between cellulose fibril surfaces. Experimental studies had shown that the structure of the adsorbed film of the strengthenhancing additive, besides the adsorbed amount, also had a role on the strength properties of the material (Eriksson 2006). In the case of the CPAM polyelectrolyte (see Fig. 11b) it was also observed that, in general lines, the bond strength also correlated well with the strength factor associated to each model. At $1 \mathrm{X}$ surface concentration the bond strength increased slightly in comparison to neat CFS, which was related to the increment also observed in the strength factor. The $2 \mathrm{X}$ and $3 \mathrm{X}$ models, which contains two and three molecules of CPAM in between the fibril surfaces respectively, gave place to a similar bond strength considering the statistical uncertainty of the results (both in the range 130-140 MPa), which was in agreement with the fact that both models exhibited also the same strength factor.

The strengthening effect of polyelectrolytes could be ascribed, as can be seen from the presented results, either to an increase in the relative bonded area between fibrils or to an increase in the number of interactions per unit area (here considered as an estimator of the specific bond strength). The presence of the polyelectrolyte molecules in the (1-10) interstices in between the microfibrils that constitute the fibril surfaces fill these void gaps and contribute to increase the $\mathrm{RBA}_{\mathrm{m}}$, i.e. the polyelectrolytes could be understood as adhesives filling the void spaces in between fibril surfaces. Besides, the polyelectrolyte molecules establish a higher number of interactions 
per unit area (density of interactions) with the cellulose surface than the cellulose with itself (compare the neat CFS model with the polyelectrolyte modified ones), which finally contribute to create a stronger interaction between the fibril surfaces. These promising results support the statement made by Lindström et al. (2005), as was cited before, and the validity of the CFS models to reproduce the expected behavior of inter-fibre joints in terms of the specific bond strength and the relative bonded area in cellulose fibre materials. Thus the CFS models become a suitable complement to other techniques for the systematic study of the effect (in qualitative terms) of chemical or physical factors on the bond strength properties of cellulosic materials. As a final comment, it should be considered that the results presented here have been obtained under some initial assumptions in order to simplify the model as a first approximation. The presented CFS models included no ionic groups in the cellulose, which would allow to the ion exchange process with polyelectrolyte molecules and would resemble in a better way with the real adsorption process, which would be expected to affect the values of measured bond strength in a certain grade. In addition, the structure of the polyelectrolyte molecules used for the modification of the CFS models was chosen with a certain DS or ionicity and a certain molecular weight just to carry out a sensitivity analysis, but not to extrapolate the results to the real system characteristics or to compare the effect between polyelectrolytes. It is also expected that these parameters would affect the results (mainly in terms of bond strength properties) in a certain grade, so if a comparison of the strengthening effect between different species of polyelectrolytes (e.g. CMC and CPAM) is desired, then the structure of the polyelectrolytes should be chose judiciously and in a more systematic way.

\section{Concluding remarks}

In our work we have developed representative models of cellulose fibril surface (CFS) as a first approximation to the study of the molecular interactions that are developed between cellulose fibres. These models were non-covalently surface modified with two types of polyelectrolytes, sodium carboxymethyl cellulose (CMC-ONa) and a cationic polyacrylamide (CPAM), in order to assess its sensitivity and representativeness towards the main factors affecting the bonding properties between cellulose fibres. From the analysis of specific pair correlation functions $(g(r))$ it was observed that the main interactions of adsorption of polyelectrolytes were due to electrostatic interactions coupled with hydrogen bonding in both type of polyelectrolytes. Our results have also shown that the polyelectrolyte molecules can interact with cellulose surfaces with no need of ion exchange, and the electrostatic interaction of polyelectrolytes could also be developed with the polarized hydroxyl groups in cellulose.

On the other hand, using steered molecular dynamics (SMD) a series of pull out simulations were carried out in order to determine the bond strength between fibril surfaces through the (100) hydrophobic surface (normal component). To this extent it was observed that the rate of change of force (the main parameter controlling the pulling force) had a clear impact on the calculated bond strength and it was seen that the higher the rate of change of force the higher the bond strength between fibril surfaces. By fixing the rate of change of force at $0.159 \mathrm{nN} \mathrm{ps}^{-1}$ the calculated bond strength for the neat CFS model (nanometer scale) was two to three orders of magnitude higher than the experimental values observed at the fibre scale (micrometer scale). We also observed that the CFS models were sensitive to the chemical modification of the surface of the fibrils conducted by polyelectrolyte adsorption and reproduced the expected behavior of inter-fibre joints with respect to the specific bond strength (measured in terms of the density of interactions) and the relative bonded area (here defined as $\mathrm{RBA}_{\mathrm{m}}$ ) in cellulose fibre materials. The proposed CFS models are thus a good first approximation to the study of the bonds between fibres at the very deep molecular level, but further improvements of these models could be done in terms of using more accurate force field potentials (such as Class II or III) or including other factors in the models such as the presence of non cellulosic cell wall components such as lignin or xyloglucans, or ionic groups in the cellulose molecules (e.g. carboxylic groups).

Acknowledgments This work has been partly granted by Spanish Ministry of Economy, Industry and Competitiveness (project RTC-2014-2817-5) and by FSE Operative Programme for Aragon (2014-2020). 


\section{References}

Alder BJ, Wainwright TE (1959) Studies in molecular dynamics. I. general method. J Chem Phys 31:459-466. https:// doi.org/10.1063/1.1730376

Batchelor WJ, Jihong H (2005) A new method for determining the relative bonded area. Tappi J 4:24-28

Bergenstråhle M, Mazeau K, Berglund LA (2008) Molecular modeling of interfaces between cellulose crystals and surrounding molecules: effects of caprolactone surface grafting. Eur Polym J 44:3662-3669. https://doi.org/10. 1016/j.eurpolymj.2008.08.029

Bergenstråhle M, Thormann E, Nordgren N, Berglund LA (2009) Force pulling of single cellulose chains at the crystalline cellulose - liquid interface: a molecular dynamics study. Langmuir 25:4635-4642. https://doi.org/ $10.1021 / 1 \mathrm{a} 803915 \mathrm{c}$

Biermann O (2001) Molecular dynamics simulation study of polyelectrolyte adsorption on cellulose surfaces. Universität Dortmund, Dortmund

Bourassa P, Bouchard J, Robert S (2013) Quantum chemical calculations of pristine and modified crystalline cellulose surfaces: benchmarking interactions and adsorption of water and electrolyte. Cellulose. https://doi.org/10.1007/ s10570-013-0150-x

Budd J, Herrington TM (1989) Surface charge and surface area of cellulose fibres. Colloids Surf 36:273-288. https://doi. org/10.1016/0166-6622(89)80243-4

Da Silva Perez D, Ruggiero R, Morais LC, Machado A, Mazeau K (2004) Theoretical and experimental studies on the adsorption of aromatic compounds onto cellulose. Langmuir 20:3151-3158. https://doi.org/10.1021/la0357817

Ding S-Y, Zhao S, Zeng Y (2014) Size, shape, and arrangement of native cellulose fibrils in maize cell walls. Cellulose 21:863-871. https://doi.org/10.1007/s10570-013-0147-5

Doblin MS, Kurek I, Jacob-Wilk D, Delmer DP (2002) Cellulose biosynthesis in plants: from genes to rosettes. Plant Cell Physiol 43:1407-1420

Enarsson L-E (2006) Polyelectrolyte adsorption on oppositely charged surfaces-conformation and adsorption kinetics. Licentiate thesis, KTH Royal Institute of Technology

Eriksson M (2006) The influence of molecular adhesion on paper strength. Doctoral thesis, KTH Royal Institute of Technology

Fadrná E, Hladečková K, Koča J (2005) Long-range electrostatic interactions in molecular dynamics: an endothelin-1 case study. J Biomol Struct Dyn 23:151-162. https://doi. org/10.1080/07391102.2005.10531229

Fischer WJ, Hirn U, Bauer W, Schennach R (2012) Testing of individual fiber-fiber joints under biaxial load and simultaneous analysis of deformation. Nord Pulp Pap Res J 27:237-244. https://doi.org/10.3183/NPPRJ-2012-27-02p237-244

Foley BL, Tessier MB, Woods RJ (2012) Carbohydrate force fields. Wiley Interdiscip Rev Comput Mol Sci 2:652-697. https://doi.org/10.1002/wcms.89

Gimåker M (2007) Influence of adsorbed polyelectrolytes and adsorption conditions on creep properties of paper sheets made from unbleached kraft pulp. Doctoral thesis, KTH Royal Institute of Technology
Hadden JA, French AD, Woods RJ (2013) Unraveling cellulose microfibrils: a twisted tale. Biopolymers 99:746-756. https://doi.org/10.1002/bip.22279

Hardy BJ, Sarko A (1996) Molecular dynamics simulations and diffraction-based analysis of the native cellulose fibre: structural modelling of the I- $\alpha$ and I- $\beta$ phases and their interconversion. Polymer 37:1833-1839. https://doi.org/ 10.1016/0032-3861(96)87299-5

Heinze T, Liebert T (2001) Unconventional methods in cellulose functionalization. Prog Polym Sci 26:1689-1762. https://doi.org/10.1016/S0079-6700(01)00022-3

Hirn U, Schennach R (2015) Comprehensive analysis of individual pulp fiber bonds quantifies the mechanisms of fiber bonding in paper. Sci Rep. https://doi.org/10.1038/ srep 10503

Hoover WG (1985) Canonical dynamics: equilibrium phasespace distributions. Phys Rev A 31:1695-1697. https://doi. org/10.1103/PhysRevA.31.1695

Ikai A, Afrin R, Sekiguchi H (2007) Pulling and pushing protein molecules by AFM. Curr Nanosci 3:17-29. https://doi.org/ 10.2174/157341307779940535

Jorgensen WL, Chandrasekhar J, Madura JD, Impey RW, Klein ML (1983) Comparison of simple potential functions for simulating liquid water. J Chem Phys 79:926-935. https:// doi.org/10.1063/1.445869

Kargl R, Mohan T, Bračič M, Kulterer M, Doliška A, StanaKleinschek K, Ribitsch V (2012) Adsorption of carboxymethyl cellulose on polymer surfaces: evidence of a specific interaction with cellulose. Langmuir 28:11440-11447. https://doi.org/10.1021/la302110a

Leach A (2001) Molecular Modelling: Principles and Applications, 2nd Edition, 2nd edn. Pearson Prentice Hall, Great Britain

Levlin J-E, Söderberg L (1999) Papermaking science and technology: pulp and paper testing. Book 17. Fapet

Li W, Huang S, Xu D, Zhao Y, Zhang Y, Zhang L (2017) Molecular dynamics simulations of the characteristics of sodium carboxymethyl cellulose with different degrees of substitution in a salt solution. Cellulose 24:3619-3633. https://doi.org/10.1007/s10570-017-1364-0

Lindström T, Wågberg L, Larsson T (2005) On the nature of joint strength in paper. A review of dry and wet strength resins used in paper manufacturing. The Pulp and Paper Fundamental Research Society Cambridge, UK, pp 457-562

Magnusson MS, Östlund S (2013) Numerical evaluation of interfibre joint strength measurements in terms of three-dimensional resultant forces and moments. Cellulose 20:1691-1710. https://doi.org/10.1007/s10570-013-9939-x

Magnusson MS, Zhang X, Östlund S (2013) Experimental evaluation of the interfibre joint strength of papermaking fibres in terms of manufacturing parameters and in two different loading directions. Exp Mech 53:1621-1634. https://doi.org/10.1007/s11340-013-9757-y

Mayo S, Olafson B, Goddard W (1990) Dreiding—a generic force-field for molecular simulations. J Phys Chem 94:8897-8909. https://doi.org/10.1021/j100389a010

Mazeau K (2011) On the external morphology of native cellulose microfibrils. Carbohydr Polym 84:524-532. https:// doi.org/10.1016/j.carbpol.2010.12.016 
Mazeau K, Vergelati C (2002) Atomistic modeling of the adsorption of benzophenone onto cellulosic surfaces. Langmuir 18:1919-1927. https://doi.org/10.1021/la010792q

Mazeau K, Wyszomirski M (2012) Modelling of Congo red adsorption on the hydrophobic surface of cellulose using molecular dynamics. Cellulose 19:1495-1506. https://doi. org/10.1007/s10570-012-9757-6

Minko S, Kiriy A, Gorodyska G, Stamm M (2002) Single flexible hydrophobic polyelectrolyte molecules adsorbed on solid substrate: transition between a stretched chain, necklace-like conformation and a globule. J Am Chem Soc 124:3218-3219. https://doi.org/10.1021/ja017767r

Miyamoto H, Umemura M, Aoyagi T, Yamane C, Ueda K, Takahashi K (2009) Structural reorganization of molecular sheets derived from cellulose II by molecular dynamics simulations. Carbohydr Res 344:1085-1094. https://doi. org/10.1016/j.carres.2009.03.014

Morfill J, Neumann J, Blank K, Steinbach U, Puchner EM, Gottschalk KE, Gaub HE (2008) Force-based analysis of multidimensional energy landscapes: application of dynamic force spectroscopy and steered molecular dynamics simulations to an antibody fragment-peptide complex. J Mol Biol 381:1253-1266. https://doi.org/10. 1016/j.jmb.2008.06.065

Nishiyama Y, Johnson GP, French AD, Forsyth VT, Langan P (2008) Neutron crystallography, molecular dynamics, and quantum mechanics studies of the nature of hydrogen bonding in cellulose I $\beta$. Biomacromol 9:3133-3140. https://doi.org/10.1021/bm800726v

Nordman LS (1957) Bonding in paper sheets. In: Fundamentals of papermaking fibres Transactions of the Cambridge Symposium

Nosé S (1984) A molecular dynamics method for simulations in the canonical ensemble. Mol Phys 52:255-268. https://doi. org/10.1080/00268978400101201

O'Sullivan A (1997) Cellulose: the structure slowly unravels. Cellulose 4:173-207. https://doi.org/10.1023/A: 1018431705579

Oehme DP, Doblin MS, Wagner J, Bacic A, Downtown MT, Gidley MJ (2015) Gaining insight into cell wall cellulose macrofibril organisation by simulating microfibril adsorption. Cellulose 22:3501-3520. https://doi.org/10.1007/ s10570-015-0778-9

Page DH (2002) The meaning of Nordman bond strength. Nord Pulp Paper Res J 17:39-44

Plimpton S (1995) Fast parallel algorithms for short-range molecular dynamics. J Comput Phys 117:1-19. https://doi. org/10.1006/jcph.1995.1039

Schmied FJ, Teichert C, Kappel L, Hirn U, Schennach R (2012) Joint strength measurements of individual fiber-fiber bonds: an atomic force microscopy based method. Rev Sci Instrum 83:073902. https://doi.org/10.1063/1.4731010

Schmied FJ, Teichert C, Kappel L, Hirn U, Bauer W, Schennach R (2013) What holds paper together: nanometre scale exploration of bonding between paper fibres. Sci Rep. https://doi.org/10.1038/srep02432

Stratton R (1993) Characterization of fiber-fiber bond strength from out-of-plane paper mechanical-properties. J Pulp Pap Sci 19:J6-J12

Stukowski A (2010) Visualization and analysis of atomistic simulation data with OVITO-the open visualization tool.
Model Simul Mater Sci Eng 18:015012. https://doi.org/10. 1088/0965-0393/18/1/015012

Su J, Garvey CJ, Holt S, Tabor RF, Winther-Jensen B, Batchelor W, Garnier G (2015) Adsorption of cationic polyacrylamide at the cellulose-liquid interface: a neutron reflectometry study. J Colloid Interface Sci 448:88-99. https:// doi.org/10.1016/j.jcis.2015.02.008

Torgnysdotter A, Wågberg L (2004) Influence of electrostatic interactions on fibre/fibre joint and paper strength. Nord Pulp Pap Res J 19:440-447. https://doi.org/10.3183/ NPPRJ-2004-19-04-p440-447

Torgnysdotter A, Kulachenko A, Gradin P, Wågberg L (2007) The link between the fiber contact zone and the physical properties of paper: a way to control paper properties. J Compos Mater 41:1619-1633. https://doi.org/10.1177/ 0021998306069875

Trejo-O'reilly J-A, Cavaille J-Y, Gandini A (1997) The surface chemical modification of cellulosic fibres in view of their use in composite materials. Cellulose 4:305-320. https:// doi.org/10.1023/A:1018452310122

Van de Steeg HGM, Cohen Stuart MA, De Keizer A, Bijsterbosch BH (1992) Polyelectrolyte adsorption: a subtle balance of forces. Langmuir 8:2538-2546. https://doi.org/10. 1021/la00046a030

Van den Akker JA (1959) Structural Aspects of Bonding. Tappi 42:940

Wagberg L (2000) Polyelectrolyte adsorption onto cellulose fibres - a review. Nord Pulp Pap Res J 15:586-597

Wang J, Somasundaran P (2005) Adsorption and conformation of carboxymethyl cellulose at solid-liquid interfaces using spectroscopic, AFM and allied techniques. J Colloid Interface Sci 291:75-83. https://doi.org/10.1016/j.jcis.2005.04.095

Wathén R (2006) Studies on fiber strength and its effect on paper properties. KCL Commun 11. Thesis dissertation, Department of Forest Products Technology, Helsinki University of Technology. https://www.semanticscholar. org/paper/Studies-on-fiber-strength-and-its-effect-onpaper-Wath\%C3\%A9n/7b7997497ef94acd16ad0c428cf 5b1688d99d52b

Watts HD, Mohamed MNA, Kubicki JD (2014) A DFT study of vibrational frequencies and 13C NMR chemical shifts of model cellulosic fragments as a function of size. Cellulose 21:53-70. https://doi.org/10.1007/s10570-013-0128-8

Winter L, Wågberg L, Ödberg L, Lindström T (1986) Polyelectrolytes adsorbed on the surface of cellulosic materials. J Colloid Interface Sci 111:537-543. https://doi.org/10. 1016/0021-9797(86)90057-3

Zhang Q, Brumer H, Agren H, Tu Y (2011) The adsorption of xyloglucan on cellulose: effects of explicit water and side chain variation. Carbohydr Res 346:2595-2602. https:// doi.org/10.1016/j.carres.2011.09.007

Zhang T, Zheng Y, Cosgrove DJ (2016) Spatial organization of cellulose microfibrils and matrix polysaccharides in primary plant cell walls as imaged by multichannel atomic force microscopy. Plant J 85:179-192. https://doi.org/10. 1111/tpj.13102

Zhao Z, Crespi VH, Kubicki JD, Cosgrove DJ, Zhong L (2014) Molecular dynamics simulation study of xyloglucan adsorption on cellulose surfaces: effects of surface hydrophobicity and side-chain variation. Cellulose 21:1025-1039. https://doi.org/10.1007/s10570-013-0041-1 